\title{
Higgs Mass Bounds from Renormalization Flow for a simple Yukawa model
}

\author{
Holger Gies,, , Clemens Gneiting, ${ }^{2}$, 田 and René Sondenheimer ${ }^{1, \text { 团 }}$ \\ ${ }^{1}$ Theoretisch-Physikalisches Institut, Friedrich-Schiller-Universität Jena, Max-Wien-Platz 1, D-07r433 Jena, Germany \\ ${ }^{2}$ Physikalisches Institut, Albert-Ludwigs-Universität Freiburg, \\ Hermann-Herder-Straße 3, D-79104 Freiburg, Germany
}

\begin{abstract}
We study the functional renormalization group flow of a Higgs-Yukawa toy model mimicking the top-Higgs sector of the standard model. This approach allows for treating arbitrary bare couplings. For the class of standard bare potentials of $\phi^{4}$-type at a given ultraviolet cut-off, we show that a finite infrared Higgs mass range emerges naturally from the renormalization group flow itself. Higgs masses outside the resulting bounds cannot be connected to any conceivable set of bare parameters in this standard-model $\phi^{4}$ class. By contrast, more general bare potentials allow to diminish the lower bound considerably. We identify a simple renormalization group mechanism for this depletion of the lower bound. If active also in the full standard model, Higgs masses smaller than the conventional infrared window do not necessarily require new physics at low scales or give rise to instability problems.
\end{abstract}

\section{INTRODUCTION}

The recent potential discovery of the standard model Higgs boson with a comparatively low mass of $m_{\mathrm{H}} \simeq$ $125 \mathrm{GeV}$ [1] has stimulated renewed interest in Higgs mass bounds within the standard model itself $[2-7]$ and beyond 8 11]. In particular, arguments based on vacuum stability [12 18] (or sufficient metastability [19 22]) appear to give rise to a lower bound for the Higgs mass [23 28]. The measured value for the mass of the discovered scalar boson is either close to or on top of the bound or might even violate the bound, depending on various other parameters, most notably the value of the top mass (in the appropriate scheme) and the strong coupling constant.

The consequences of the true Higgs mass satisfying or violating the bound can be rather dramatic, ranging from measured constraints on the underlying UV theory structure, via an upper bound for the scale of new physics to the prediction of the decay of the universe as we know it. Therefore, a thorough understanding of Higgs mass bounds within the standard model is clearly mandatory.

Even though typical computations of mass bounds are often done with perturbative (RG-resummed) methods, the problem is generically nonperturbative. This is obvious for the upper Higgs mass bound - the so-called unitarity or triviality bound - which is, in principle, related to a strongly coupled Higgs sector in the UV. In perturbation theory, this becomes manifest from the vicinity to the Landau pole, indicating the failure of perturbation theory 11 But also the lower bound involves nonperturbative information for two reasons: first, the prediction

\footnotetext{
* holger.gies@uni-jena.de

$\dagger$ clemens.gneiting@physik.uni-freiburg.de

$\ddagger$ rene.sondenheimer@uni-jena.de

1 In fact, the upper bound is often motivated from the requirement that the standard model per definitionem should be describable within perturbation theory in the UV. Since this is if at all an aesthetic but not a physical criterion, we rely on the criterion of triviality in the present work.
}

of infrared (IR) quantities such as Higgs and top masses involve a proper description of threshold effects. These are nonperturbative, as such mass scales are related to the couplings. Second, an investigation of stability issues requires the computation of a full effective potential for arbitrary field amplitudes.

In a series of works, Higgs mass bounds have therefore recently been studied within lattice quantum field theory both for a simple $\mathbb{Z}_{2}$ Higgs-Yukawa model [29 31], as well as for a Higgs-Yukawa model more similar to and significant for the standard model [31, 32. In particular, the lower Higgs mass bound arises from the mere criterion of starting from a physically meaningful bare UV theory on the lattice. No reference to low-energy stability issues had to be made, and no indications for an instability have been observed. Most prominently, the simulations of 33 , 34] essentially rule out or put strong constraints on the existence of a fourth flavor generation for the measured Higgs boson mass; similar conclusions have been drawn from analytic considerations [35].

In the present work, we revisit the Higgs mass bounds by analytic means using the functional renormalization group (RG). Within a consistent systematic derivative expansion, the functional RG provides for a tool to analyze the problem nonperturbatively and allows to estimate errors of the approximation scheme. In order to concentrate on the basic mechanisms for the mass bounds, we confine ourselves to the simple $\mathbb{Z}_{2}$ HiggsYukawa model, as it avoids intricate questions arising from the gauge-Higgs interplay in the full standard model [36, 37], while at the same time maintaining the standard model property that no Goldstone bosons arise in the broken phase. First functional RG studies of Higgs mass bounds have already been performed in [38, 39].

In the present work, we particularly concentrate on the influence of generic UV actions on the Higgs mass bounds. In fact, we find a rather substantial influence of the precise form of the bare scalar potential on the lower bound of the Higgs boson. At first sight, this seems to be at odds with common wisdom of renormalizable field theories that IR observables should be independent of 
the details of the microscopic UV theory. This statement (formulated under suitable mild assumptions) is, of course, left untouched by our work. However, the main point is that the notion of a Higgs mass bound is strictly speaking not a pure IR observable. Higgs mass bounds are typically formulated as a function of the UV cutoff $\Lambda$, i.e., $m_{\mathrm{H} \text {,bound }}=m_{\mathrm{H} \text {,bound }}(\Lambda)$. Hence, in order to quantify this dependence, we have to make certain assumptions about the system at and near the cutoff. This includes the choice of a regularization scheme, specifying the details of the UV regularization at the cutoff; in this sense, part of the scheme-dependence of the Higgs mass bounds is actually physical. And this includes dynamical properties of the flow near the cutoff which can be rather strongly influenced by the bare theory. Quantitatively, we find that rather mild modifications of the bare potential can have a significant impact on the lower Higgs mass bound.

This article is organized as follows: in Sect. II] we briefly introduce our simple toy model. Section III summarizes the concepts of the functional RG applied to this model and presents the resulting flow equations. As a warm-up, a simple mean-field analysis already illustrating many of the properties of the Higgs mass bounds is given in Sect. IV] Incidentally, these mean-field properties do actually not require the functional RG framework, but could equally well be derived within a large- $N$ type of reasoning. Our main results based on the nonperturbative RG flow equations are summarized in Sect. $\mathrm{V}$

\section{II. $\mathbb{Z}_{2}$-SYMMETRIC HIGGS-YUKAWA TOY MODEL}

Many of the fluctuation-induced features of Higgs mass bounds in the standard model can already be studied in a greatly simplified model involving a Dirac fermion flavor $\psi$ (the top quark) and a real scalar boson $\phi$. The model is defined by the Euclidean classical action

$$
S=\int d^{4} x\left[\frac{1}{2}\left(\partial_{\mu} \phi\right)^{2}+\frac{\bar{m}}{2} \phi^{2}+\frac{\bar{\lambda}}{8} \phi^{4}+\bar{\psi} i \not \partial \psi+i \bar{h} \phi \bar{\psi} \psi\right] .
$$

For later purposes, we allow the top quark to appear in $N_{\mathrm{f}}$ flavor copies. We use $N_{\mathrm{f}}$ merely as an ordering parameter of the calculation, but not as a physical parameter mimicking the generations of the standard model. For quantitative statements, we will use $N_{\mathrm{f}}=1$. The model is invariant under a discrete "chiral" symmetry,

$$
\psi \rightarrow \mathrm{e}^{i \frac{\pi}{2} \gamma_{5}} \psi, \quad \bar{\psi} \rightarrow \bar{\psi} \mathrm{e}^{i \frac{\pi}{2} \gamma_{5}}, \quad \phi \rightarrow-\phi,
$$

which protects the fermions against acquiring a direct mass term. Since the symmetry is only discrete, its spontaneous breaking owing to a nonzero expectation value for the scalar field $v=\langle\phi\rangle$ does not give rise to massless Goldstone bosons. This feature mimics the property of the standard model that the Goldstone modes are eaten by the massive electroweak gauge bosons.
The quantum theory corresponding to Eq. (11) has to be defined with a finite ultraviolet (UV) cutoff $\Lambda$ which, together with a specified regularization prescription, remains an implicit physical parameter of the theory. This is because triviality inhibits an ultraviolet extension to arbitrarily high scales while keeping the physical lowenergy parameters fixed [40]; in perturbation theory, this feature is reflected by the existence of a Landau pole in the running coupling.

Solving the so defined quantum theory provides for a mapping from the microscopic bare parameters $\bar{m}^{2}, \bar{\lambda}, \bar{h}, \Lambda$ and possibly further RG irrelevant bare couplings to the set of physical parameters which are given by the top mass $m_{\text {top }}$, the Higgs mass $m_{\mathrm{H}}$, the vacuum expectation value $v$ and still the cutoff $\Lambda$. These physical parameters are directly related to renormalized couplings in the quantum effective action, such as the renormalized Yukawa coupling $h$, see below, and the effective potential $U(\rho)$, where $\rho=\frac{\phi^{2}}{2}$. Denoting the minimum of the effective potential by $\rho_{0}$, we identify

$$
v=Z_{\phi}^{1 / 2}\langle\phi\rangle=\sqrt{2 Z_{\phi} \rho_{0}}, m_{\mathrm{top}}^{2}=v^{2} h^{2}, m_{\mathrm{H}}^{2}=v^{2} \frac{U^{\prime \prime}\left(\rho_{0}\right)}{Z_{\phi}^{2}},
$$

where the wave function renormalization $Z_{\phi}$ is introduced below. In this work, we consider the vacuum expectation value and the top mass as given, $v \simeq 246 \mathrm{GeV}$ and $m_{\text {top }} \simeq$ $173 \mathrm{GeV} 2$ Furthermore, choosing a fixed cutoff $\Lambda$ leaves only $m_{\mathrm{H}}$ as a free parameter which becomes a function of the whole set of microscopic bare parameters.

Constraints on the Higgs mass are now obtained if the region of attainable Higgs masses is bounded for any given combination of bare parameters. These bare parameters are essentially unconstrained, as they are provided by a yet unknown underlying microscopic theory (UV completion). Only a stable bare scalar potential bounded from below is required in order to facilitate a meaningful definition of the quantum theory. In the present work, we start with the standard class of initial bare $\bar{\lambda} \phi^{4}$ potentials. UV stability then implies that $\bar{\lambda} \geq 0$ for this class of potentials. We then extend our considerations to more general potentials. For instance, also a negative $\bar{\lambda}$ is permitted if the potential is stabilized for large $\phi$, e.g., by positive $\phi^{6}, \phi^{8}, \ldots$ terms in the bare potential. We emphasize that these higher-order terms cannot be excluded by referring to renormalizability criteria. This is because we consider them to be present in

\footnotetext{
${ }^{2}$ We use here the value for the top mass measured by kinematically reconstructing its decay products and comparing these to Monte Carlo simulations. For Higgs mass bounds, actually the pole mass is considered to be the appropriate quantity, which could significantly differ from the experimentally quoted value [26]. As a rule of thumb, an uncertainty of $\sim 1 \mathrm{GeV}$ in the top mass leads to a $\pm 2 \mathrm{GeV}$ variation of the lower Higgs mass bound for large cutoffs $\Lambda$. In any case, quantitative results of the present toy model should anyway only be considered as an illustrative example.
} 
the microscopic UV potential at a fixed (possibly physical) UV cutoff $\Lambda$. Presently no experiment can impose relevant constraints on such terms which could arise from an underlying UV completion of the standard model. Renormalizability rather tells us that the IR is dominated by the power-counting "renormalizable" operators in the standard model, provided that the UV theory starts near the perturbative Gaußian fixed point.

\section{RENORMALIZATION FLOW}

As an alternative to the functional-integral definition of continuum quantum field theory, we use a differential formulation provided by the functional RG. A convenient version is given by the flow equation for the effective average action $\Gamma_{k}$, which interpolates between the bare action $\Gamma_{k=\Lambda}=S$ at the UV cutoff $\Lambda$ and the full quantum effective action $\Gamma=\Gamma_{k=0}$ [41]. The latter corresponds to the generator of fully-dressed proper vertices. The variation of the effective action with respect to the scale $k$ is given by the Wetterich equation

$k \partial_{k} \Gamma_{k} \equiv \partial_{t} \Gamma_{k}=\frac{1}{2} \operatorname{STr}\left[\left(\partial_{t} R_{k}\right)\left(\Gamma_{k}^{(2)}+R_{k}\right)^{-1}\right], t=\ln \frac{k}{\Lambda}$.

Here, $\Gamma_{k}^{(2)}$ denotes the second functional derivative with respect to the fluctuating fields $\Phi=(\phi, \psi, \bar{\psi})$, and the super-trace also includes a minus sign for the fermions. The regulator $R_{k}$ in the denominator is chosen such that it suppresses IR modes below the scale $k$, and its derivative $k \partial_{k} R_{k}$ establishes ultraviolet (UV) finiteness; as a consequence, the flow of $\Gamma_{k}$ is dominated by fluctuations with momenta $p^{2} \simeq k^{2}$, implementing the concept of smooth momentum-shell integrations, for reviews see 42 48].

As we are working with an explicit finite cutoff $\Lambda$, also the choice of the regularization scheme strictly speaking belongs to the definition of the model. This scheme is here specified in terms of the regulator function $R_{k}$, more precisely in terms of the regulator shape functions $r\left(p^{2} / k^{2}\right), r_{\mathrm{F}}\left(p^{2} / k^{2}\right)$ introduced in the appendix. From the viewpoint of the model definition, these shape functions determine how the modes are physically cut off in the UV. Since a change of the regularization scheme such as a change of the shape functions can be mapped onto a change of the initial conditions for the bare couplings, we keep the regulator fixed in the present work and vary the bare couplings.

In addition to perturbative expansions, nonperturbative approximation schemes can be devised for the flow equation. Systematic and consistent expansion schemes which do not rely on a perturbative coupling ordering are, for instance, the vertex expansion or the derivative expansion.

In this work, we study the renormalization flow of the
Yukawa system nonperturbatively within the following truncation based on the derivative expansion:

$\Gamma_{k}=\int_{x}\left[\frac{Z_{\phi, k}}{2}\left(\partial_{\mu} \phi\right)^{2}+U_{k}(\rho)+Z_{\psi, k} \bar{\psi} i \not \partial \psi+i \bar{h}_{k} \phi \bar{\psi} \psi\right]$

where $\rho=\frac{1}{2} \phi^{2}$, and the potential $U_{k}$ generally includes arbitrary powers of the field. In fact, the accuracy of the derivative expansion for scalar theories has been verified quantitatively in many contexts. Here, we actively study its convergence by comparing leading-order (LO) results (obtained for $Z_{\phi, k}=1, Z_{\psi, k}=1$ ) to next-to-leading order (NLO) results. We find no signatures of a failure of this expansion even at comparatively strong coupling, see below.

Inserting this ansatz (5) into the flow equation (4) provides us with the RG flows of $\bar{h}_{k}, U_{k}$ and the wave function renormalizations $Z_{\phi, k}$ and $Z_{\psi, k}$; the latter flows will be followed in terms of the anomalous dimensions

$$
\eta_{\phi}=-\partial_{t} \ln Z_{\phi, k}, \quad \eta_{\psi}=-\partial_{t} \ln Z_{\psi, k}
$$

The flow equation for the effective potential reads

$$
\begin{array}{r}
\partial_{t} U_{k}=2 v_{d} k^{d}\left[l_{0}^{d}\left(k^{-2} Z_{\phi, k}^{-1}\left[2 \rho U_{k}^{\prime \prime}+U_{k}^{\prime}\right] ; \eta_{\phi}\right)\right. \\
\left.-N_{\mathrm{f}} d_{\gamma} l_{0}^{(F) d}\left(2 k^{-2} Z_{\psi, k}^{-2} \bar{h}_{k}^{2} \rho ; \eta_{\psi}\right)\right],
\end{array}
$$

where the primes denote derivatives with respect to $\rho$, and $v_{d}^{-1}=2^{d+1} \pi^{d / 2} \Gamma(d / 2)$. For generality, we work in $d$ dimensions and with a $d_{\gamma}$ dimensional representation of the Dirac algebra. We will later specialize to $d=4$ and $d_{\gamma}=4$. The threshold functions $l_{0}^{d}$ and $l_{0}^{(F) d}$ arise from the integration over the loop momentum and carry the non-universal regulator dependence. For any physically admissible regulator, they approach finite constants for vanishing argument and decrease to zero for large first argument, describing the decoupling of massive modes; details can be found in Appendix A

It is useful to introduce renormalized fields

$$
\tilde{\phi}=Z_{\phi, k}^{1 / 2} \phi, \quad \tilde{\psi}=Z_{\psi, k}^{1 / 2} \psi
$$

as well as the dimensionless renormalized $\mathrm{Z}_{2}$ invariant quantity

$$
\tilde{\rho}=Z_{\phi, k} k^{2-d} \rho .
$$

The dimensionless renormalized Yukawa coupling is defined by

$$
h_{k}^{2}=Z_{\phi, k}^{-1} Z_{\psi, k}^{-2} k^{d-4} \bar{h}_{k}^{2}
$$

and the dimensionless potential simply is:

$$
u_{k}=U_{k} k^{-d}
$$

The flow of $u_{k}$ for fixed $\tilde{\rho}$ is given by 


$$
\partial_{t} u_{k}=-d u_{k}+\left(d-2+\eta_{\phi}\right) \tilde{\rho} u_{k}^{\prime}+2 v_{d}\left[l_{0}^{d}\left(u_{k}^{\prime}+2 \tilde{\rho} u_{k}^{\prime \prime} ; \eta_{\phi}\right)-N_{\mathrm{f}} d_{\gamma} l_{0}^{(F) d}\left(2 \tilde{\rho} h_{k}^{2} ; \eta_{\psi}\right)\right],
$$

where primes now denote derivatives with respect to $\tilde{\rho}$. The flow of the Yukawa coupling is of the form

$$
\begin{aligned}
\partial_{t} h_{k}^{2}= & {\left[\eta_{\phi}+2 \eta_{\psi}+d-4\right] h_{k}^{2}+8 h_{k}^{4} v_{d} l_{1,1}^{(F B) d}\left(\omega_{1}, \omega_{2} ; \eta_{\psi}, \eta_{\phi}\right) } \\
& -\left[48 \kappa_{k} u_{k}^{\prime \prime}\left(\kappa_{k}\right)+32 \kappa_{k}^{2} u_{k}^{\prime \prime \prime}\left(\kappa_{k}\right)\right] h_{k}^{4} v_{d} l_{1,2}^{(F B) d}\left(\omega_{1}, \omega_{2} ; \eta_{\psi}, \eta_{\phi}\right)-32 h_{k}^{6} \kappa_{k} v_{d} l_{2,1}^{(F B) d}\left(\omega_{1}, \omega_{2} ; \eta_{\psi}, \eta_{\phi}\right)
\end{aligned}
$$

with

$$
\omega_{1}=2 \kappa_{k} h_{k}^{2}, \quad \omega_{2}=u_{k}^{\prime}\left(\kappa_{k}\right)+2 \kappa_{k} u_{k}^{\prime \prime}\left(\kappa_{k}\right),
$$

and $\kappa_{k}=\tilde{\rho}_{\min }$ denotes the minimum of the potential; i.e., if $\kappa_{k} \neq 0$ then $u_{k}^{\prime}\left(\kappa_{k}\right)=0$. Finally, the anomalous dimensions are determined by

$$
\begin{gathered}
\eta_{\phi}=8 \frac{v_{d}}{d}\left[\kappa_{k}\left[3 u_{k}^{\prime \prime}\left(\kappa_{k}\right)+2 \kappa_{k} u_{k}^{\prime \prime \prime}\left(\kappa_{k}\right)\right]^{2} m_{4,0}^{d}\left(2 \kappa_{k} u_{k}^{\prime \prime}\left(\kappa_{k}\right)+u_{k}^{\prime}\left(\kappa_{k}\right), 0 ; \eta_{\phi}\right)\right. \\
\left.\quad+N_{\mathrm{f}} d_{\gamma} h_{k}^{2}\left[m_{4}^{(F) d}\left(2 \kappa_{k} h_{k}^{2} ; \eta_{\psi}\right)-2 \kappa_{k} h_{k}^{2} m_{2}^{(F) d}\left(2 \kappa_{k} h_{k}^{2} ; \eta_{\psi}\right)\right]\right], \\
\eta_{\psi}=8 h_{k}^{2} \frac{v_{d}}{d} m_{1,2}^{(F B) d}\left(2 \kappa_{k} h_{k}^{2}, 2 \kappa_{k} u_{k}^{\prime \prime}\left(\kappa_{k}\right)+u_{k}^{\prime}\left(\kappa_{k}\right) ; \eta_{\psi}, \eta_{\phi}\right),
\end{gathered}
$$

where the threshold functions are again discussed in Appendix $\mathrm{A}$. These flow equations can be compared to those of similar investigations in the literature [49 51] within different physical contexts. Once the flow equations have been solved for suitable initial conditions, we can read off the fully renormalized long-range quantities in the limit $k \rightarrow 0$. For instance, the physical quantities defined in Eq. (3) require the renormalized Yukawa coupling $h=h_{k \rightarrow 0}$ and the wave function renormalization $Z_{\phi}=Z_{\phi, k \rightarrow 0}$. The renormalized vacuum expectation value is obtained from $v^{2}=\lim _{k \rightarrow 0} 2 k^{2} \kappa_{k}$.

\section{MEAN-FIELD ANALYSIS}

Let us first perform a mean-field analysis, corresponding to a one-loop approximation of the effective potential including fermion as well as boson fluctuations, while keeping the wave function renormalizations and the Yukawa coupling fixed,

$$
Z_{\phi, k}, Z_{\psi, k} \rightarrow 1, \quad h_{k} \rightarrow h_{\Lambda}
$$

The mean-field effective potential $U^{\mathrm{MF}}$ could, of course, be calculated directly from a Gaußian approximation of the generating functional, yielding the standard log detformula. Nevertheless, we derive it from the flow equation, since it provides direct access to the use of an arbitrarily shaped regulator function, which can be used to model the physical UV cutoff mechanism.

The standard mean-field (MF) approximation is equivalent to the large- $N_{\mathrm{f}}$ approximation, taking only fermionic fluctuations into account. The corresponding mean-field effective potential is obtained from the flow equation (7) by integrating the fermion contributions $\sim N_{\mathrm{f}}$ from $k=\Lambda$ to 0 , while keeping the potential on the right-hand side fixed at $U_{k} \rightarrow U_{\Lambda}$. We obtain for the mean-field effective potential

$$
\begin{aligned}
U_{k}^{\mathrm{MF}}(\rho)= & U_{\Lambda}(\rho) \\
& +\frac{N_{\mathrm{f}} d_{\gamma}}{2} \int_{p} \ln \left(\frac{p^{2}\left(1+r_{\mathrm{F}}\left(p^{2} / \Lambda^{2}\right)\right)^{2}+2 \bar{h}_{\Lambda}^{2} \rho}{p^{2}\left(1+r_{\mathrm{F}}\left(p^{2} / k^{2}\right)\right)^{2}+2 \bar{h}_{\Lambda}^{2} \rho}\right),
\end{aligned}
$$

where $\int_{p}=\int \frac{d^{d} p}{(2 \pi)^{d}}$. The extended mean-field (EMF) approximation is obtained by including also the scalar fluctuations on the same Gaußian level. Introducing the abbreviation

$$
M_{\Lambda}^{2}(\rho)=U_{\Lambda}^{\prime}(\rho)+2 \rho U_{\Lambda}^{\prime \prime}(\rho)
$$

we find,

$$
\begin{aligned}
U_{k}^{\mathrm{EMF}}(\rho)= & U_{k}^{\mathrm{MF}}(\rho) \\
& -\frac{1}{2} \int_{p} \ln \left[\frac{p^{2}\left(1+r\left(p^{2} / \Lambda^{2}\right)\right)+M_{\Lambda}^{2}(\rho)}{p^{2}\left(1+r\left(p^{2} / k^{2}\right)\right)+M_{\Lambda}^{2}(\rho)}\right] .
\end{aligned}
$$

Whereas the mean-field approximation becomes exact in the strict large- $N_{\mathrm{f}}$ limit, no such anchoring to an exact limit is known for the extended-mean-field approximation. Moreover, further subtleties arise in the extendedmean-field case from convexity violations and complex solutions for the potential as discussed in [52]. These subtleties of the extended mean-field approximation are however irrelevant for the nonperturbative functional RG solution discussed below. Hence, we will mainly stay within the standard mean-field approximation in the following for the purpose of illustration.

For both approximations, the momentum integration can be done analytically for a suitable choice of the regulator shape functions $r(x), r_{\mathrm{F}}(x)$. For instance, for the linear regulator (cf. App. A we obtain in the limit $k \rightarrow 0$ and in $d=4$ dimensions (where $\bar{h}_{\Lambda}=h_{\Lambda}$ ) 


$$
\begin{gathered}
U^{\operatorname{EMF}}(\rho)=U_{\Lambda}(\rho)+\frac{1}{64 \pi^{2}}\left\{\left[M_{\Lambda}^{2}(\rho)-M_{\Lambda}^{2}(0)-2 N_{\mathrm{f}} d_{\gamma} h_{\Lambda}^{2} \rho\right] \Lambda^{2}+4 N_{\mathrm{f}} d_{\gamma} h_{\Lambda}^{4} \rho^{2} \ln \frac{\Lambda^{2}+2 h_{\Lambda}^{2} \rho}{2 h_{\Lambda}^{2} \rho}\right. \\
\left.-M_{\Lambda}^{4}(\rho) \ln \frac{\Lambda^{2}+M_{\Lambda}^{2}(\rho)}{M_{\Lambda}^{2}(\rho)}+M_{\Lambda}^{4}(0) \ln \frac{\Lambda^{2}+M_{\Lambda}^{2}(0)}{M_{\Lambda}^{2}(0)}\right\},
\end{gathered}
$$

where we have normalized $U^{\operatorname{EMF}}(\rho)$ such that $U^{\mathrm{EMF}}(0)=0$. In the following we will show that Eq. (21) can be used to illustrate the appearance of a lower bound for the Higgs mass.

\section{A. Bare potentials of $\phi^{4}$-type}

Let us confine ourselves to bare potentials of $\phi^{4}$-type,

$$
U_{\Lambda}(\rho)=m_{\Lambda}^{2} \rho+\frac{\lambda_{\Lambda}}{2} \rho^{2} .
$$

For a given UV cutoff $\Lambda$, two out of the three bare parameters $m_{\Lambda}^{2}, \lambda_{\Lambda}, h_{\Lambda}$ can be fixed by fixing the top mass and the vacuum expectation value; more precisely, fixing $h_{\Lambda}=m_{\mathrm{top}} / v$ and determining $m_{\Lambda}^{2}$ from the transcendental equation

$$
U^{\mathrm{EMF} \prime}\left(\rho_{0}=v^{2} / 2\right)=0,
$$

leaves us with the Higgs mass as a function of the bare scalar coupling, $m_{\mathrm{H}}=m_{\mathrm{H}}\left(\lambda_{\Lambda}\right)$. In the standard meanfield approximation, it is easy to see that $m_{\mathrm{H}}=m_{\mathrm{H}}\left(\lambda_{\Lambda}\right)$ increases monotonically with $\lambda_{\Lambda}$, therefore a lower bound on the Higgs mass is obtained from the lowest possible value of $\lambda_{\Lambda}$, which is $\lambda_{\Lambda, \min }=0$ for potentials of the form of Eq. (22). (In the extended mean-field approximation, the same conclusion holds unless $\lambda_{\Lambda}$ approaches the strong-coupling value $\lambda_{\Lambda} \rightarrow \frac{8}{3} h_{\Lambda}^{2}$ where an EMF-artifact induces singular behavior).

Equation (23) can easily be solved numerically. For an analytical estimate, let us stay within the mean-field approximation and keep only the terms $\sim N_{\mathrm{f}}$. Determining $m_{\Lambda}^{2}$ from the condition $U^{\mathrm{MF} \prime}\left(\rho_{0}=v^{2} / 2\right)=0$ for fixed values of $m_{\mathrm{top}}$ and $v$, we find (setting $N_{\mathrm{f}}=1, d_{\gamma}=4$ )

$$
\begin{aligned}
m_{\Lambda}^{2}\left(\Lambda, \lambda_{\Lambda}\right)= & -\frac{\lambda_{\Lambda}}{2} v^{2}+\frac{h_{\Lambda}^{2}}{8 \pi^{2}} \Lambda^{2} \\
& -\frac{h_{\Lambda}^{4} v^{2}}{8 \pi^{2}}\left[2 \ln \left(1+\frac{\Lambda^{2}}{m_{\text {top }}^{2}}\right)-\frac{\Lambda^{2}}{\Lambda^{2}+m_{\text {top }}^{2}}\right] .
\end{aligned}
$$

This fixes the effective mean-field potential as a function of $\lambda_{\Lambda}$ and $\Lambda$, yielding the Higgs mass

$$
\begin{aligned}
m_{\mathrm{H}}^{2}\left(\Lambda, \lambda_{\Lambda}\right)= & v^{2} U^{\mathrm{MF} \prime \prime}\left(v^{2} / 2\right) \\
= & \frac{m_{\mathrm{top}}^{4}}{4 \pi^{2} v^{2}}\left[2 \ln \left(1+\frac{\Lambda^{2}}{m_{\mathrm{top}}^{2}}\right)-\frac{3 \Lambda^{4}+2 m_{\mathrm{top}}^{2} \Lambda^{2}}{\left(\Lambda^{2}+m_{\mathrm{top}}^{2}\right)^{2}}\right] \\
& +v^{2} \lambda_{\Lambda} .
\end{aligned}
$$

This renders explicit that the lower bound for UV potentials of the form of Eq. (22) is given by $m_{\mathrm{H}}\left(\Lambda, \lambda_{\Lambda}=0\right)$.

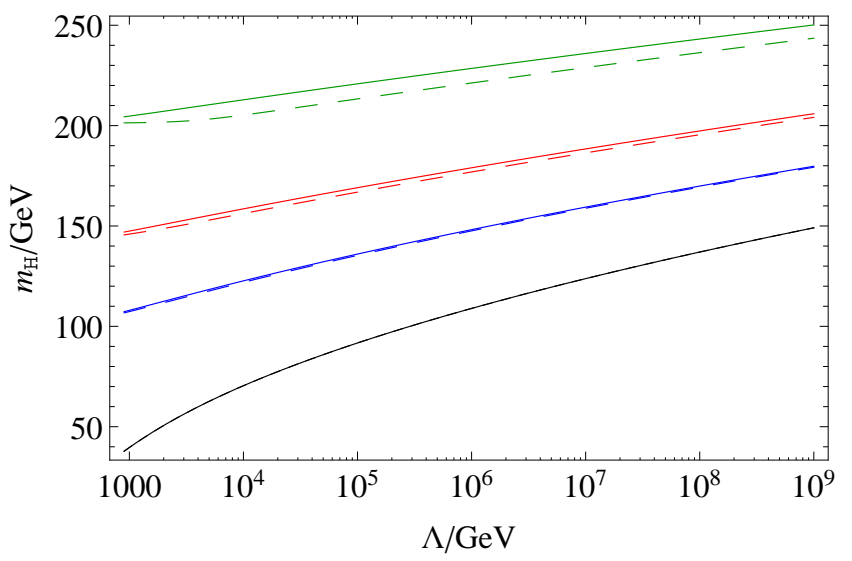

FIG. 1. Extended mean-field analysis of the lower bound for the Higgs mass $m_{\mathrm{H}}$ versus the UV cutoff $\Lambda$, based on a bare potential $U_{\Lambda}$ of $\phi^{4}$-type for $N_{\mathrm{f}}=1$. For an initial potential which is flat apart from a mass term $U_{\Lambda}=\frac{1}{2} m_{\Lambda}^{2} \phi^{2}$, the fermionic fluctuations drive the Higgs mass to a finite minimal value. The solid lines correspond to standard meanfield theory accounting only for top fluctuations, cf. Eq. (25), whereas the dashed lines also include scalar fluctuations on the Gaußian level (extended mean-field). The four different line sets correspond to increasing values of the initial $\phi^{4}$ coupling of $\lambda_{\Lambda}=0, \frac{1}{6}, \frac{1}{3}, \frac{2}{3}$ from bottom to top.

The mean-field analysis performed here gives a first insight into how lower bounds for the Higgs mass follow from the mapping from bare to renormalized quantities. It also exemplifies that the mere existence of a lower bound on the Higgs mass for bare potentials of $\phi^{4}$-type is essentially a consequence of top fluctuations that drive the curvature of the effective potential at its nontrivial minimum to finite values. This statement will also hold on the nonperturbative level. We plot the mean-field results for the Higgs mass as a function of $\Lambda$ for various values of $\lambda_{\Lambda}$ in Fig. 1 as solid lines.

The plot also shows corrections from bosonic fluctuations as described by extended mean-field theory $U^{\mathrm{EMF}}(\rho)$ as dashed lines for the same values of $\lambda_{\Lambda}$. We observe that scalar fluctuations tend to decrease the Higgs mass values. This agrees with the fact that scalar fluctuations drive the effective potential towards the symmetric regime, thus depleting also the curvature near the minimum. However, the lower bound of the Higgs mass remains unaffected by the scalar fluctuations, because the scalar field is non-interacting for $\lambda_{\Lambda}=0$ in the EMF approximation. 


\section{B. Generalized bare potentials}

The lower Higgs mass bound determined above arises from the fact that the values for the bare quartic coupling $\lambda_{\Lambda}$ are bounded from below. This is necessary in order to start with a well-defined theory in the UV for our confined bare potentials (22) of $\phi^{4}$-type. Such a restriction on the bare potential is typically also required in perturbation theory because higher-order operators are perturbatively non-renormalizable. By contrast, the Wetterich equation provides us with a nonperturbative tool, so we can study also the influence of RG irrelevant higher-order operators on the flow of the effective average action. Alternatively, this could also be studied with perturbative methods in an effective-theory approach.

In the following we address the question how modifications $\Delta U_{\Lambda}(\rho)$ of the quartic bare potential can exert an influence on the lower Higgs mass bound. The bare potential can in principle be an arbitrary function of the scalar field. The only constraint which we impose is that the potential is bounded from below in order to start from a well-defined quantum field theory at the cutoff. We emphasize that no further experimental constraints exist. The simplest extention of the standard potential has an additional operator of the form $\phi^{6}$.

$$
\begin{aligned}
U_{\Lambda}(\rho) & =m_{\Lambda}^{2} \rho+\frac{\lambda_{\Lambda}}{2} \rho^{2}+\Delta U_{\Lambda}(\rho) \\
& =m_{\Lambda}^{2} \rho+\frac{\lambda_{\Lambda}}{2} \rho^{2}+\frac{\lambda_{3, \Lambda}}{6 \Lambda^{2}} \rho^{3} .
\end{aligned}
$$

Again, in the mean-field case Eq. (23) can be solved explicitly for $m_{\Lambda}^{2}$, yielding the Higgs mass as a function of $\lambda_{\Lambda}$ and $\lambda_{3, \Lambda}$ for a given cutoff, $m_{\mathrm{H}}=m_{\mathrm{H}}\left(\lambda_{\Lambda}, \lambda_{3, \Lambda}\right)$. With $\lambda_{3, \Lambda}$ positive we can study a wider range of values for the bare quartic coupling. The Higgs mass reads

$$
m_{\mathrm{H}}^{2}\left(\Lambda, \lambda_{\Lambda}, \lambda_{3, \Lambda}\right)=\frac{m_{\mathrm{top}}^{4}}{4 \pi^{2} v^{2}}\left[2 \ln \left(1+\frac{\Lambda^{2}}{m_{\mathrm{top}}^{2}}\right)-\frac{3 \Lambda^{4}+2 m_{\mathrm{top}}^{2} \Lambda^{2}}{\left(\Lambda^{2}+m_{\mathrm{top}}^{2}\right)^{2}}\right]+v^{2} \lambda_{\Lambda}+\frac{v^{4}}{2 \Lambda^{2}} \lambda_{3, \Lambda} .
$$

Obviously, we are able to construct a theory with a Higgs mass below the previous lower bound if the contribution of the term $\sim \lambda_{\Lambda}$ for $\lambda_{\Lambda}<0$ exceeds that of the positive term $\sim \lambda_{3, \Lambda}$

The same mechanism works in the extended mean-field analysis but there it requires a solution to the transcendental Eq. (23) in order to determine $m_{\Lambda}^{2}$. A numerical solution is plotted in Fig. 2 for different values of $\lambda_{\Lambda}$ and $\lambda_{3, \Lambda}$. Furthermore, we have checked that for the given masses no additional minimum appears in the effective potential besides the one at $v=246 \mathrm{GeV}$.

Let us finally remark that upper bounds cannot meaningfully be studied in the mean-field approximation; this is because "RG improvement" is necessary to observe the nonperturbative approach to triviality (reflected by the Landau-pole behavior within RG-improved perturbation theory).

\section{NONPERTURBATIVE HIGGS MASS BOUNDS}

The mean-field approximation has turned out to be remarkably accurate by direct comparison with nonperturbative lattice simulations for the present model [29, 30]. As lattice simulations are typically limited as far as the separation of the UV scale from the physical scales is concerned, a nonperturbative continuum analysis of beyond mean-field theory seems indispensable in order to appropriately account for scalar fluctuations and the mutual back-reactions between fermionic and scalar fluctuations

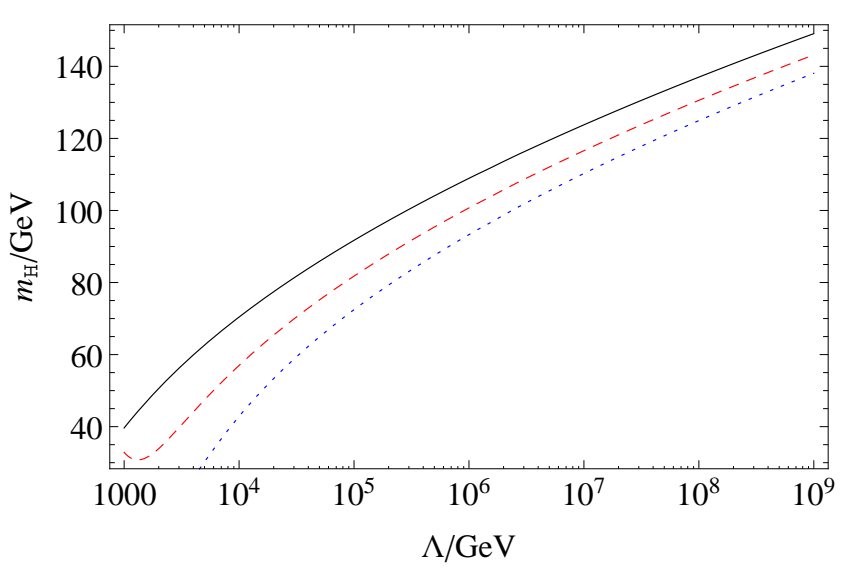

FIG. 2. Extended mean-field analysis of the lower bound for the Higgs mass $m_{\mathrm{H}}$ versus the UV cutoff $\Lambda$, based on a bare potential $U_{\Lambda}$ of $\phi^{6}$-type for $N_{\mathrm{f}}=1$. We have plotted the lower bound in the $\phi^{4}$ theory $\left(\lambda_{\Lambda}=\lambda_{3, \Lambda}=0\right)$ as solid black line. Theories with bare couplings $\lambda_{\Lambda}=-\frac{1}{30}$ and $\lambda_{3, \Lambda}=\frac{2}{3}$ are depicted as red dashed line, and $\lambda_{\Lambda}=-\frac{1}{15}$ and $\lambda_{3, \Lambda}=2$ as blue dotted line.

on a wide range of scales.

For the solution of the flow equations, we use the formulation in terms of dimensionless renormalized quantities as introduced in Sect. III To leading-order in the derivative expansion, we solve the flow equations for the effective potential $u_{k}$ and for the Yukawa coupling $h_{k}$. At next-to-leading order, we include the wave-function 
renormalizations $\eta_{\phi}$ and $\eta_{\psi}$.

Since we are mainly interested in the properties of the effective potential near its minimum, we use a polynomial expansion of the potential. The stability and convergence of this expansion will be checked explicitly. In the symmetric regime (SYM) where the minimum of the potential occurs at $\kappa_{k}=0$, we use the truncated expansion

$$
u_{k}=\sum_{n=1}^{N_{\mathrm{p}}} \frac{\lambda_{n}}{n !} \tilde{\rho}^{n},
$$

such that the potential is parameterized by $N_{\mathrm{p}}$ couplings $\lambda_{n}$ (the mass term is related to $\lambda_{1}$ and we identify the $\phi^{4}$ interaction as $\lambda \equiv \lambda_{2}$ ). In the symmetry-broken regime (SSB), we instead use

$$
u_{k}=\sum_{n=2}^{N_{\mathrm{p}}} \frac{\lambda_{n}}{n !}\left(\tilde{\rho}-\kappa_{k}\right)^{n} .
$$

The flows of $\lambda_{1}, \ldots, \lambda_{N_{\mathrm{p}}}$ (SYM), or $\kappa_{k}, \lambda_{2}, \ldots \lambda_{N_{\mathrm{p}}}$ (SSB), can directly be derived from Eq. (12).

For small bare scalar coupling $\lambda_{\Lambda} \equiv \lambda_{2, \Lambda}$, a physical flow typically starts in the SYM regime. Near the electroweak scale, fermionic fluctuations drive the system into the SSB regime at a scale $k_{\mathrm{SSB}}$, where we have to switch from the SYM flow to the SSB flow. Here, a nonzero minimum builds up, inducing masses for the fermions and the Higgs scalar. This leads to a decoupling of the modes, and the flow freezes out completely; i.e., all right-hand sides of the flow equations go to zero for $k \rightarrow 0$. For large bare scalar coupling $\lambda_{\Lambda}$, the physical flow starts already in the SSB regime with a small value for $\kappa_{k}$. The flow can still run over many scales until $\kappa_{k}$ grows large near the electroweak scale, implying again the decoupling of all modes.

\section{A. $\phi^{4}$-type bare potentials}

Let us again start with the restricted class of bare potentials of $\phi^{4}$-type,

$$
u_{\Lambda}=\lambda_{1, \Lambda} \tilde{\rho}+\frac{\lambda_{\Lambda}}{2} \tilde{\rho}^{2},
$$

where $\lambda_{1, \Lambda} \equiv m_{\Lambda}^{2} / \Lambda^{2}$ for a wave function renormalization $Z_{\phi, \Lambda}=1$. For a given cutoff $\Lambda$, the flow equations map the bare parameters $m_{\Lambda}^{2}, \lambda_{\Lambda}, h_{\Lambda}$ onto the physical parameters $v, m_{\mathrm{top}}, m_{\mathrm{H}}$. In practice, we tune $m_{\Lambda}^{2}$ to establish the correct vacuum expectation value $v \simeq 246 \mathrm{GeV}$ for a given cutoff $\Lambda$. This is, in fact, a fine-tuning problem, corresponding to the problem of separating the scale hierarchies in the standard model. At the same time, $h_{\Lambda}$ is varied until the flow ends at the right value of $m_{\text {top }}$. This leaves us with the Higgs mass as a function of $\lambda_{\Lambda}$ for a given cutoff $\Lambda, m_{\mathrm{H}}=m_{\mathrm{H}}\left(\Lambda, \lambda_{\Lambda}\right)$, where $\lambda_{\Lambda}$ is allowed to be an a priori arbitrary non-negative real number for the class of bare potentials (30).

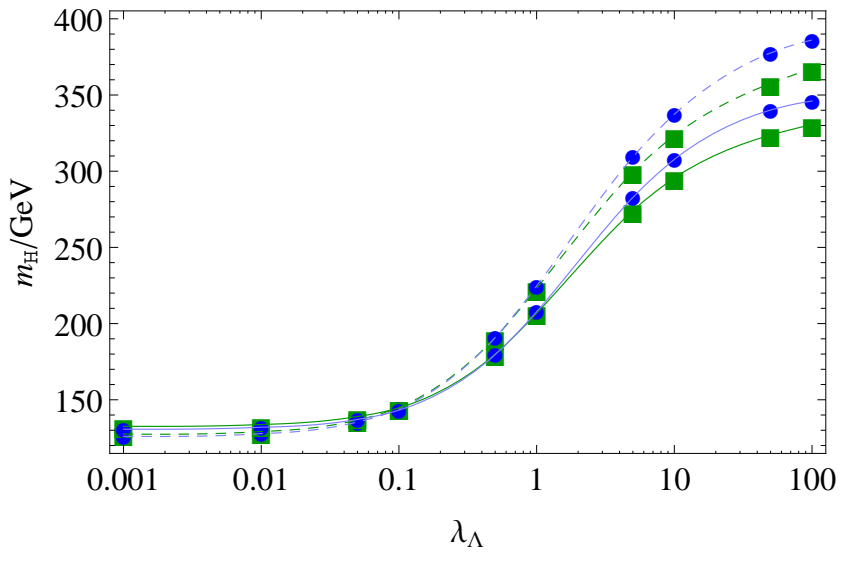

FIG. 3. Higgs mass values versus the bare scalar coupling $\lambda_{\Lambda}$ for a cutoff $\Lambda=10^{7} \mathrm{GeV}$. The dashed lines denote the results within LO derivative expansion; the NLO deviates from the LO result by at most $10 \%$ for large coupling, demonstrating the satisfactory convergence of the derivative expansion. Also the convergence of the polynomial expansion is shown: green lines with squares are obtained within the lowest nontrivial order with $N_{\mathrm{p}}=2$, blue lines with circles denote the $N_{\mathrm{p}}=4$ result; even higher orders $N_{P}=6,8$ show no further deviation from the $N_{\mathrm{p}}=4$ curves.

In Fig. 3, we depict this function $m_{\mathrm{H}}\left(\lambda_{\Lambda}\right)$ for a cutoff $\Lambda=10^{7} \mathrm{GeV}$ for various approximations. For $\lambda_{\Lambda} \lesssim 0.01$, the Higgs mass becomes rather independent of $\lambda_{\Lambda}$ approaching its lower bound. This observation is in perfect agreement with lattice simulations [29, 30, 32 34]. For larger bare coupling $\lambda_{\Lambda}$, the Higgs mass increases and approaches a regime of saturation for $\lambda_{\Lambda} \gg 1$. This is reminiscent to RG-improved perturbation theory, where the bare coupling hits the Landau pole $\lambda_{\Lambda} \rightarrow \infty$ already at a finite cutoff $\Lambda$.

Whereas the Landau pole in perturbation theory in the first place signals the breakdown of the perturbative expansion, our truncation of the RG flow does neither rely on perturbative ordering nor require a weak coupling. Instead, our derivative expansion is organized in terms of field operators with increasing number of derivatives. In order to check the convergence of this expansion, we can compare the results for the Higgs mass to leading order (LO) and next-to-leading order (NLO) in this expansion. To leading order, we drop the running of the kinetic terms in Eq. (5) by setting the anomalous dimensions to zero, $\eta_{\psi, \phi} \rightarrow 0$. The resulting Higgs masses are plotted as dashed lines in Fig. 3. We observe that the difference to the NLO result (solid lines) is rather small for the lower Higgs mass bound for $\lambda_{\Lambda} \rightarrow 0$; even for the largest accessible couplings, we observe a maximum deviation of $10 \%$, confirming that the derivative expansion constitutes a satisfactory approximation for our purpose for the whole range from weak to strong coupling.

Furthermore, we study the convergence of the polynomial expansion of the scalar potential in Fig. 3, To low- 


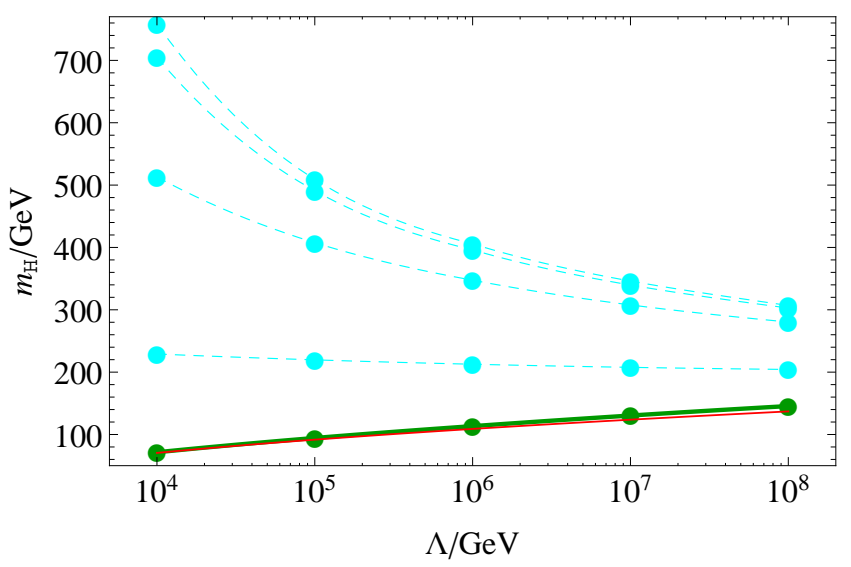

FIG. 4. Higgs mass bounds versus cutoff $\Lambda$. The thick green/solid line denotes the lower bound for the Higgs mass derived within the class of bare $\phi^{4}$ potentials. The thin $\mathrm{red} /$ solid line shows the lower bound as derived within meanfield approximation. The turquois/dashed lines mark upper bounds if the bare scalar coupling is allowed to start maximally from $\lambda_{\Lambda}=1,10,50,100$ from bottom to top, respectively. An artificial restriction to the perturbative domain $\lambda_{\Lambda} \lesssim 1$ underestimates the upper bound by a factor $\gtrsim \mathcal{O}(1)$.

est nontrivial order $N_{\mathrm{p}}=2$ (green lines with squares), we obtain already a complete picture of the physics of Higgs mass bounds. For the next order $N_{\mathrm{p}}=4$ (blue lines with circles), though the upper Higgs mass bound is already approached for smaller bare couplings $\lambda_{\Lambda}$, the value of the upper bound changes by at most $5 \%$. For even higher orders, the corresponding results lie on top of the $N_{\mathrm{p}}=4$ curves. Within our numerical accuracy we find no significant difference for $N_{\mathrm{p}}=4,6,8$.

In Fig. 4, we show the resulting Higgs mass bounds, arising within the class of $\phi^{4}$ bare potentials. The thick solid/green line characterizes the lower bound resulting from the RG flow for a wide range of cutoffs $\Lambda=10^{4} \ldots 10^{8} \mathrm{GeV}$. Also shown is the lower bound as derived within the mean-field approximation in the previous section (thin solid/red line), which neglects the running of the Yukawa coupling, of the anomalous dimension, and RG improvement of the scalar potential. In the full flow, we observe nontrivial cancelations among these terms, such that the mean-field result represents a surprisingly good approximation over a wide range of cutoff scales. The turquois/dashed lines depict upper bounds for the Higgs mass for bare couplings $\lambda_{\Lambda}=1,10,50,100$, respectively. In particular, we find that if we limited ourselves to a perturbative domain, choosing $\lambda_{\Lambda}=1$, we would artificially underestimate the upper bound by a factor $\gtrsim \mathcal{O}(1)$.

\section{B. Generalized bare potentials}

Let us now study extensions of the initial bare potential beyond the $\phi^{4}$-type. Motivated by the results of the mean-field approximation, we concentrate on potentials with a negative $\lambda_{2, \Lambda}$ where the UV stability is guaranteed by a positive $\lambda_{3} \phi^{6}$. It is possible to construct bare potentials which give rise to Higgs masses below the lower bound within the class of $\phi^{4}$ bare potentials, similar to the mean-field approach. Fig. 5 shows the lower bound within $\phi^{4}$ theory (black solid line) in comparison to Higgs mass values for an example flow which starts with $\lambda_{2, \Lambda}=-0.1$ and $\lambda_{3, \Lambda}=3$ in the UV (red solid line). This example clearly illustrates that the lower bound within $\phi^{4}$-like initial potentials does no longer hold, if higher dimensional operators are also permitted.

This phenomenon can be understood from the RG flow itself: first we note that in both cases ( $\phi^{4}$-like as well as the beyond- $\phi^{4}$ example above) the flow starts in the symmetric regime. In the beyond- $\phi^{4}$ example, the quartic coupling $\lambda_{2}$ runs quickly to positive values, whereas $\lambda_{3}$ becomes very small as is expected in the vicinity of the Gaußian fixed point. As a consequence, this particular system flows back into the class of $\phi^{4}$-type potentials. The decisive difference, however, is that the scale $k_{\mathrm{GFP}}$ where the system is again near the Gaußian fixed point is now lower than the initial UV scale $\Lambda$. Loosely speaking, some "RG time" is required to run from the beyond- $\phi^{4}$ form of the potential back to the $\phi^{4}$ Gaußian type.

From another viewpoint, the RG flow can map an initial bare action with $\lambda_{2}<0$ and $\lambda_{3}>0$ at an initial UV scale $\Lambda$ to a theory with $\lambda_{2} \geq 0$ and $\lambda_{3} \approx 0$ at a smaller scale $k_{\mathrm{GFP}}<\Lambda$. Therefore, the red curve (beyond- $\phi^{4}$ ) in Fig. 5 can also be viewed as a horizontally displaced version of the black curve ( $\phi^{4}$-like) to effectively larger cutoff values. We emphasize that the present example has neither been specifically designed or fine-tuned, nor does it represent an exhaustive study of admissible initial potentials. A wide range of beyond- $\phi^{4}$ potentials initiating the flow at $\Lambda$ leads to Higgs masses below the bound of the $\phi^{4}$-type class. Still, the mechanism observed above starting from stable potentials with $\lambda_{2}<0$ and globally stabilizing higher-order terms appears rather generic. We have also checked for more involved initial conditions that the results for the Higgs masses do not change for higher-order $N_{P} \geq 4$ polynomial expansions of the scalar potential.

In fact, the influence of higher dimensional operators has also been studied in recent lattice simulations in a chiral Higgs-Yukawa model [34], by adding a positive $\lambda_{3} \phi^{6}$ term to the bare potential. No lowering of the Higgs mass bound has been observed in this study. This is indeed in agreement with our observations, because merely adding this term has barely any effect on the Higgs mass bound and rather leads to an increase of the Higgs mass. Our mechanism for lowering the mass bound works particularly well for initial potentials with $\lambda_{2}<0$. In other words, the $\lambda_{2}<0$ deformation requires a comparatively 


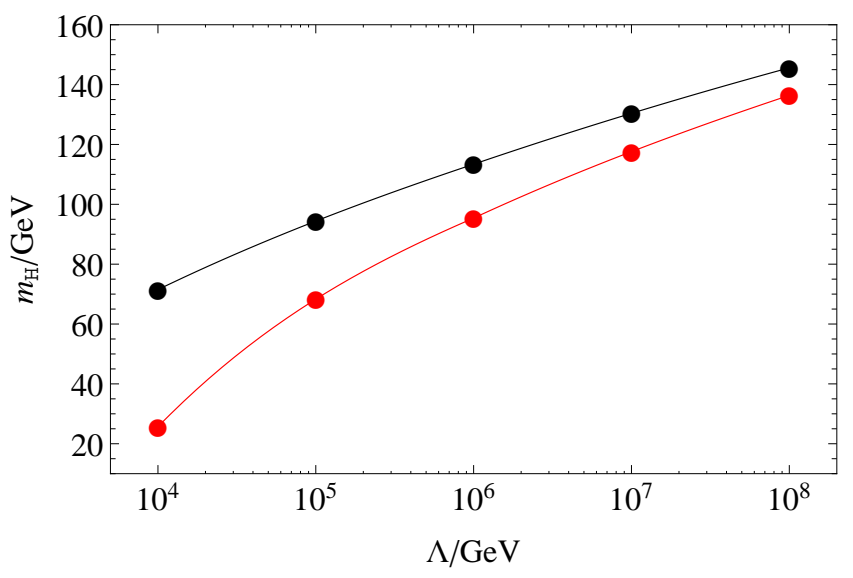

FIG. 5. Higgs mass versus cutoff $\Lambda$. The black line denotes the lower bound for the Higgs mass derived within the class of bare $\phi^{4}$ potentials. The red line shows how we can construct Higgs masses below the lower bound by giving up the restriction to quartic bare potentials. The masses are derived for $\lambda_{2}=-0.1$ and $\lambda_{3}=3$.

long RG time to run the potential back to the $\phi^{4}$ Gaußian type. A lattice study with such (or even more general) bare potentials could hence put our mechanism to test.

Having put the significance of the lower bound of the Higgs mass derived for $\phi^{4}$-type bare potentials into perspective, let us address the issue of stability: while the standard approach to vacuum stability in the present simple model based on RG-improved perturbation theory has been questioned by lattice simulations [29, 30] and functional RG methods [38] (in turn critically assessed by [53]), a full stability analysis would require to follow the RG flow of arbitrary physically admissible initial potentials. In particular, the $\mathrm{RG}$ evolution of potentials with multiple local minima would have to be dealt with quantitatively. While this is indeed possible with appropriate numerical solvers [39, 49, 54 56], we here confine ourselves to the validity region of the polynomial expansion of the effective potential about a local minimum.

Since high-order polynomials typically have multiple local minima, we have to estimate the radius of convergence of our expansion in field space. A new local minimum showing up within this convergence region could then be interpreted as a signature of instability. If such minima only occur outside the convergence radius, we consider them as an artifact of the polynomial expansion.

A rough estimate for the radius of convergence is given by comparing the quotients of successive couplings $\lambda_{n} / \lambda_{n+1}$ for large $n$ in the infrared. In practice we solve the system of coupled differential equations for $N_{P}=20$, switching back to dimensionful quantities at a scale where the flows are frozen out, e.g. $U_{k}=u_{k} k^{4}=$ $\sum_{n} a_{n}\left(Z_{\phi} \rho-v^{2} / 2\right)^{n}$ with $a_{n}=\frac{\lambda_{n}}{n !} k^{4-2 n}$, and computing the dimensionful radius of convergence by comparing $\frac{a_{n}(k)}{a_{n+1}(k)}$ for $k \rightarrow 0$. The results expressed in units of

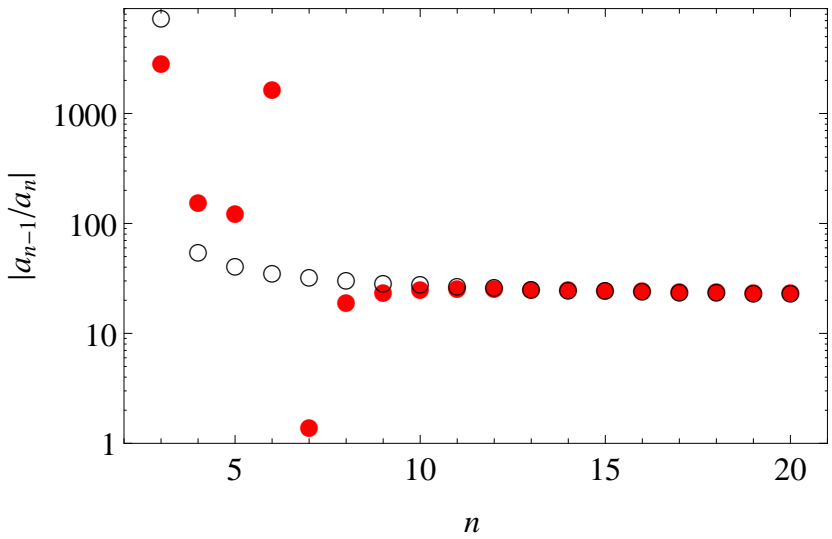

FIG. 6. Estimate for the radius of convergence in units of $10^{3} \mathrm{GeV}^{2}$ of the polynomial expansion of the effective potentials in terms of the absolute values of the ratios of expansion coefficients. The red filled circles are derived for a theory which starts at $\Lambda=10^{7} \mathrm{GeV}$ with all couplings set to zero apart from the mass term. The black empty circles are for the case $\Lambda=10^{7} \mathrm{GeV}$ and $\lambda_{2}=1$ and $\lambda_{n}=0(n \geq 3)$.

$10^{3} \mathrm{GeV}$ for various initial conditions are plotted in Fig. 6.

Our primary observation is that this estimate for the radius of convergence appears to stabilize at a universal value rather independent of the chosen initial conditions. The resulting value near $\simeq 23000 \mathrm{GeV}^{2}$ is of the order of the vacuum expectation value $v^{2} / 2=30258 \mathrm{GeV}^{2}$ for large $n$. We still observe a slight drift in our data even at high order, which might be due to the fact that the inner region of the effective potential owing to its convexity cannot be resolved within a polynomial expansion as a matter of principle. Restricting the field amplitudes to values of the order of the ratio of the highest couplings in the truncation, $Z_{\phi} \rho_{\max } \simeq\left(v^{2} / 2\right)+\left|\frac{a_{N_{P}-1}}{a_{N_{P}}}\right|$, we find in all studied cases that the effective potential is a convex monotonically rising function in the outer region $(\phi>$ $v$ ). No evidence for an instability within this radius of convergence is found.

These observations agree with solutions of the RG flow for the full effective potential beyond the polynomial expansion as worked out in [39] using pseudo-spectral methods (Chebyshev expansion). Both methods lead to equivalent results for both, the Higgs mass bounds for $\phi^{4}$-type initial potentials as well as the absence of any indication for an instability.

\section{CONCLUSIONS}

We have determined Higgs mass bounds in a simple Higgs-Yukawa toy model sharing some similarities with the standard model Higgs-top-quark sector. Our study is based on the functional renormalization group which can keep track of threshold phenomena, has better access to strong coupling regimes and automatically accounts for 
"RG improvement".

In agreement with the standard literature, the existence of an upper Higgs mass bound is a consequence of triviality of the scalar sector. As such, it is inherently non-universal. In this work, we have also emphasized the non-universality of the lower Higgs mass bound. In addition to the regularization scheme dependence which the lower bound shares with the upper bound in any UV incomplete theory, we have discovered that the lower mass bound can depend sensitively on the microscopic details of the bare effective potential for the Higgs field.

This observation does not contradict Wilsonian renormalizability arguments stating that IR observables should be independent of the details of the UV theory. The reason is that a Higgs mass bound given in the form $m_{\mathrm{H} \text {,bound }}=m_{\mathrm{H} \text {, bound }}(\Lambda)$ as a function of the UV cutoff $\Lambda$ does not constitute a pure IR observable. By contrast, it should be understood as a mapping of initial conditions at the microscopic UV scale onto the set of possible IR observables. As the UV initial conditions are typically not accessible by low energy measurements, they are unconstraint. A statement about Higgs mass bounds therefore often goes along with (typically only implicit) constraints on the UV initial conditions, i.e., bare actions or bare potentials.

In the conventional discussions of Higgs mass bounds, the IR measured observables are taken from experiment and the RG flow is run to higher scales. This procedure lacks any control over RG irrelevant operators, as their influence on the IR observables is exponentially small. Therefore, their high-energy behavior is simply ignored or implicitly fixed by computational recipes such as RGimproved perturbation theory. Latest results along this line of reasoning show that the measured mass of the Higgs boson is close to the "vacuum stability" bound or even in the "metastable region" (with the biggest uncertainty arising from the exact value of the top mass, to be specified in an appropriate scheme) [25 28]. From this viewpoint, the fact that the Higgs mass together with the whole standard model is close to a phase transition is a remarkable result of the $\mathrm{LHC}$, requiring an explanation of this "near-criticality" property [28]. Since this runningup of the perturbative RG cannot access the large field regime, where a new vacuum is expected to occur, a full resolution of this near-criticality puzzle either requires nonperturbative complements or even calls for beyondstandard-model explanations.

Our results offer a different viewpoint: as we have hardly any information about the bare action at an initial scale $\Lambda$, bounds on particle masses can only arise from the mapping of all admissible bare initial conditions onto the IR observables as is provided by the RG. Of course, the resulting bounds will depend on the criteria of $a d$ missibility which we may impose. In this work, we have demonstrated that strict Higgs mass bounds arise if we restrict the initial conditions to $\phi^{4}$-type potentials. We emphasize, however, that this restriction is somewhat arbitrary: it cannot be justified by Wilsonian renormaliz- ability arguments, as they simply do not apply to bare actions. Hence, if we lift this artificial restriction, we can easily discover initial conditions that lead to Higgs masses substantially smaller than the Higgs mass bound within the $\phi^{4}$ class. This is already the case for initial potentials with comparatively small higher-order operators. Nonperturbatively large deformations of the initial potential are not required.

From this viewpoint, the near-criticality property of the standard model remains nevertheless remarkable, as it may provide for a first handle on the microscopic action at some high (GUT-like or Planck) scale that has to emerge from an underlying theory ("a UV completion"). The top-down analog of this reasoning has been used in a model with asymptotically safe gravity that predicted the value of the Higgs mass [57] (see also [58]), based on the fact that asymptotically safe gravity interactions are likely to put the Higgs mass onto its "conventional" lower bound. Already earlier, arguments for putting the standard model onto this conventional lower bound lead to similar predictions [59].

By contrast, if the Higgs mass turns out to lie below this conventional lower bound, this may not be a sufficient reason for concern regarding vacuum stability or metastability. Stability might simply be provided by higher-order operators in the initial bare action. Rather generically, we find that models with a negative $\lambda_{2, \Lambda}$ being stabilized by higher-order operators yield Higgs mass values below the conventional lower bound. Of course, the presence and magnitude of these higher order operators eventually has to be explained by a (more) UV complete underlying theory. In fact, models with a negative $\lambda_{2, \Lambda}$ have recently been discussed from a string-theory perspective [60]. A UV complete example for models with a potentially smaller Higgs mass has recently been given within pure quantum field theory in the context of an asymptotically safe gauged Higgs-Yukawa model [61].

\section{ACKNOWLEDGMENTS}

We thank Tobias Hellwig, Karl Jansen, Stefan Lippoldt, Axel Maas, Jan Pawlowski and Luca Zambelli for interesting and enlightening discussions. HG and RS acknowledge support by the DFG under grants GRK1523, Gi 328/5-2 (Heisenberg program).

\section{Appendix A: Threshold functions}

In this work, we use the linear regulator which is optimized for the present truncation [62]. For the bosonic modes, this regulator is given by

$$
R_{k}(p)=Z_{\phi, k} p^{2} r\left(p^{2} / k^{2}\right)=Z_{\phi, k}\left(k^{2}-p^{2}\right) \theta\left(k^{2}-p^{2}\right) .
$$

The corresponding chirally symmetric fermionic regulator $R_{k}(p)=Z_{\psi, k} \not p r_{\mathrm{F}}\left(p^{2} / k^{2}\right)$ is chosen such that $p^{2}(1+$ 
$r)=p^{2}\left(1+r_{\mathrm{F}}\right)^{2}$. For reasons of completeness, we list the threshold functions appearing in the main text, which can be analytically computed for the linear regulator as a result of the corresponding momentum integrations:

$$
\begin{aligned}
& l_{n}^{d}\left(\omega ; \eta_{\phi}\right)=\frac{2\left(\delta_{n, 0}+n\right)}{d} \frac{1-\frac{\eta_{\phi}}{d+2}}{(1+\omega)^{n+1}} \\
& l_{0}^{(F) d}\left(\omega ; \eta_{\psi}\right)=\frac{2\left(\delta_{n, 0}+n\right)}{d} \frac{1-\frac{\eta_{\psi}}{d+1}}{(1+\omega)^{n+1}}, \\
& l_{n_{1}, n_{2}}^{(F B) d}\left(\omega_{1}, \omega_{2} ; \eta_{\psi}, \eta_{\phi}\right)=\frac{2}{d} \frac{1}{\left(1+\omega_{1}\right)^{n_{1}}} \frac{1}{\left(1+\omega_{2}\right)^{n_{2}}} \\
& \times\left[\frac{n_{1}\left(1-\frac{\eta_{\psi}}{d+1}\right)}{1+\omega_{1}}+\frac{n_{2}\left(1-\frac{\eta_{\phi}}{d+2}\right)}{1+\omega_{2}}\right], \\
& m_{n_{1}, n_{2}}^{d}\left(\omega_{1}, \omega_{2} ; \eta_{\phi}\right)=\frac{1}{\left(1+\omega_{1}\right)^{n_{1}}\left(1+\omega_{2}\right)^{n_{2}}}, \\
& m_{2}^{(F) d}\left(\omega ; \eta_{\psi}\right)=\frac{1}{(1+\omega)^{4}}, \\
& m_{4}^{(F) d}\left(\omega ; \eta_{\psi}\right)=\frac{1}{(1+\omega)^{4}}+\frac{1-\eta_{\psi}}{d-2} \frac{1}{(1+\omega)^{3}} \\
& -\left(\frac{1-\eta_{\psi}}{2 d-4}+\frac{1}{4}\right) \frac{1}{(1+\omega)^{2}}, \\
& m_{n_{1}, n_{2}}^{(F B) d}\left(\omega_{1}, \omega_{2} ; \eta_{\psi}, \eta_{\phi}\right)=\frac{1-\frac{\eta_{\phi}}{d+1}}{\left(1+\omega_{1}\right)^{n_{1}}\left(1+\omega_{2}\right)^{n_{2}}} \text {. }
\end{aligned}
$$

These threshold functions agree with those given in [49].
[1] G. Aad et al. [ATLAS Collaboration], Phys. Lett. B 716, 1 (2012) arXiv:1207.7214 [hep-ex]]; S. Chatrchyan et al. [CMS Collaboration], Phys. Lett. B 716, 30 (2012) arXiv:1207.7235 [hep-ex]].

[2] L. Maiani, G. Parisi and R. Petronzio, Nucl. Phys. B 136, 115 (1978).

[3] N. V. Krasnikov, Yad. Fiz. 28, 549 (1978).

[4] M. Lindner, Z. Phys. C 31, 295 (1986).

[5] G. Altarelli and G. Isidori, Phys. Lett. B 337, 141 (1994).

[6] B. Schrempp and M. Wimmer, Prog. Part. Nucl. Phys. 37, 1 (1996) hep-ph/9606386.

[7] T. Hambye and K. Riesselmann, Phys. Rev. D 55, 7255 (1997) hep-ph/9610272.

[8] N. Cabibbo, L. Maiani, G. Parisi and R. Petronzio, Nucl. Phys. B 158, 295 (1979).

[9] J. R. Espinosa and M. Quiros, Phys. Lett. B 279, 92 (1992); Phys. Lett. B 302, 51 (1993) hep-ph/9212305; Phys. Lett. B 353, 257 (1995) hep-ph/9504241.

[10] C. -S. Chen and Y. Tang, JHEP 1204, 019 (2012) arXiv:1202.5717 [hep-ph]].

[11] O. Lebedev, Eur. Phys. J. C 72, 2058 (2012) arXiv:1203.0156 [hep-ph]].

[12] I. V. Krive and A. D. Linde, Nucl. Phys. B 117, 265 (1976).

[13] P. Q. Hung, Phys. Rev. Lett. 42, 873 (1979).

[14] A. D. Linde, Phys. Lett. B 92, 119 (1980).

[15] H. D. Politzer and S. Wolfram, Phys. Lett. B 82, 242 (1979) [Erratum-ibid. 83B, 421 (1979)].

[16] M. Sher, Phys. Rept. 179, 273 (1989).

[17] M. Lindner, M. Sher and H. W. Zaglauer, Phys. Lett. B 228, 139 (1989).
[18] C. Ford, D. R. T. Jones, P. W. Stephenson and M. B. Einhorn, Nucl. Phys. B 395, 17 (1993) hep-lat/9210033.

[19] P. B. Arnold, Phys. Rev. D 40, 613 (1989).

[20] M. Sher, Phys. Lett. B 317, 159 (1993) [Addendum-ibid. B 331, 448 (1994)] hep-ph/9307342.

[21] B. Bergerhoff, M. Lindner and M. Weiser, Phys. Lett. B 469, 61 (1999) hep-ph/9909261.

[22] G. Isidori, G. Ridolfi and A. Strumia, Nucl. Phys. B 609 , 387 (2001) hep-ph/0104016.

[23] J. Ellis, J. R. Espinosa, G. F. Giudice, A. Hoecker and A. Riotto, Phys. Lett. B 679, 369 (2009) arXiv:0906.0954 [hep-ph]].

[24] J. Elias-Miro, J. R. Espinosa, G. F. Giudice, G. Isidori, A. Riotto and A. Strumia, Phys. Lett. B 709, 222 (2012) arXiv:1112.3022 [hep-ph]].

[25] G. Degrassi, S. Di Vita, J. Elias-Miro, J. R. Espinosa, G. F. Giudice, G. Isidori and A. Strumia, JHEP 1208, 098 (2012) arXiv:1205.6497 [hep-ph]].

[26] S. Alekhin, A. Djouadi and S. Moch, Phys. Lett. B 716, 214 (2012) arXiv:1207.0980 [hep-ph]].

[27] I. Masina, arXiv:1209.0393 [hep-ph].

[28] D. Buttazzo, G. Degrassi, P. P. Giardino, G. F. Giudice, F. Sala, A. Salvio and A. Strumia, arXiv:1307.3536 [hep$\mathrm{ph}]$.

[29] K. Holland and J. Kuti, Nucl. Phys. Proc. Suppl. 129, 765 (2004) hep-lat/0308020.

[30] K. Holland, Nucl. Phys. Proc. Suppl. 140, 155 (2005) hep-lat/0409112.

[31] Z. Fodor, K. Holland, J. Kuti, D. Nogradi and C. Schroeder, PoS LAT 2007, 056 (2007) arXiv:0710.3151 [hep-lat]]. 
[32] P. Gerhold and K. Jansen, JHEP 0709, 041 (2007) arXiv:0705.2539 [hep-lat]]; JHEP 0710, 001 (2007) arXiv:0707.3849 [hep-lat]]; JHEP 0907, 025 (2009) arXiv:0902.4135 [hep-lat]]; JHEP 1004, 094 (2010) arXiv:1002.4336 [hep-lat]].

[33] P. Gerhold, K. Jansen and J. Kallarackal, JHEP 1101, 143 (2011) arXiv:1011.1648 [hep-lat]]; PoS LATTICE 2010, 051 (2010) [PoS ICHEP 2010, 367 (2010)] arXiv:1010.6005 [hep-lat]].

[34] J. Bulava, K. Jansen and A. Nagy, Phys. Lett. B 723, 95 (2013) arXiv:1301.3416 [hep-lat]]; PoS ConfinementX , 276 (2012); J. Bulava, P. Gerhold, A. Nagy, J. Kallarackal and K. Jansen, PoS LATTICE 2012, 054 (2012) arXiv:1301.3701 [hep-lat]].

[35] A. Djouadi and A. Lenz, Phys. Lett. B 715, 310 (2012) arXiv:1204.1252 [hep-ph]].

[36] J. Frohlich, G. Morchio and F. Strocchi, Nucl. Phys. B 190, 553 (1981).

[37] A. Maas, arXiv:1205.6625 [hep-lat]; A. Maas and T. Mufti, arXiv:1211.5301 [hep-lat].

[38] V. Branchina and H. Faivre, Phys. Rev. D 72, 065017 (2005) hep-th/0503188; V. Branchina, H. Faivre and V. Pangon, J. Phys. G 36, 015006 (2009) arXiv:0802.4423 [hep-ph]].

[39] C. Gneiting, diploma thesis, Heidelberg (2005).

[40] K. G. Wilson and J. B. Kogut, Phys. Rept. 12, 75 (1974); M. Luscher and P. Weisz, Nucl. Phys. B 295, 65 (1988); Nucl. Phys. B 318, 705 (1989); A. Hasenfratz, K. Jansen, C. B. Lang, T. Neuhaus and H. Yoneyama, Phys. Lett. B 199, 531 (1987); U. M. Heller, H. Neuberger and P. M. Vranas, Nucl. Phys. B 399, 271 (1993) arXiv:hep-lat/9207024; D. J. E. Callaway, Phys. Rept. 167, 241 (1988); O. J. Rosten, JHEP 0907, 019 (2009) arXiv:0808.0082 [hep-th]].

[41] C. Wetterich, Phys. Lett. B 301, 90 (1993).

[42] J. Berges, N. Tetradis and C. Wetterich, Phys. Rept. 363, 223 (2002), hep-ph/0005122.

[43] K. Aoki, Int. J. Mod. Phys. B 14, 1249 (2000).

[44] J. M. Pawlowski, Ann. Phys. 322, 2831 (2007), hep-th/0512261.
[45] H. Gies, Lect. Notes Phys. 852, $287 \quad$ (2012) hep-ph/0611146.

[46] B. Delamotte, Lect. Notes Phys. 852, 49 (2012) cond-mat/0702365 [COND-MAT]].

[47] P. Kopietz, L. Bartosch and F. Schutz, Lect. Notes Phys. 798, 1 (2010).

[48] J. Braun, J. Phys. G 39, 033001 (2012), arXiv:1108.4449.

[49] F. Hofling, C. Nowak and C. Wetterich, Phys. Rev. B 66, 205111 (2002) cond-mat/0203588.

[50] H. Gies and M. M. Scherer, Eur. Phys. J. C 66, 387 (2010) arXiv:0901.2459 [hep-th]].

[51] J. Braun, H. Gies and D. D. Scherer, Phys. Rev. D 83, 085012 (2011) arXiv:1011.1456 [hep-th]].

[52] E. J. Weinberg and A. -q. Wu, Phys. Rev. D 36, 2474 (1987).

[53] M. B. Einhorn and D. R. T. Jones, JHEP 0704, 051 (2007) hep-ph/0702295 [HEP-PH]].

[54] J. A. Adams, J. Berges, S. Bornholdt, F. Freire, N. Tetradis and C. Wetterich, Mod. Phys. Lett. A 10, 2367 (1995) hep-th/9507093.

[55] O. Bohr, B. J. Schaefer and J. Wambach, Int. J. Mod. Phys. A 16, 3823 (2001) hep-ph/0007098.

[56] T. Fischbacher and F. Synatschke-Czerwonka, Comput. Phys. Commun. 184, 1931 (2013) arXiv:1202.5984 [physics.comp-ph]].

[57] M. Shaposhnikov and C. Wetterich, Phys. Lett. B 683 , 196 (2010) arXiv:0912.0208 [hep-th]].

[58] F. Bezrukov, M. Y. .Kalmykov, B. A. Kniehl and M. Shaposhnikov, JHEP 1210, 140 (2012) arXiv:1205.2893 [hep-ph]].

[59] C. D. Froggatt and H. B. Nielsen, Phys. Lett. B 368, 96 (1996) hep-ph/9511371.

[60] A. Hebecker, A. K. Knochel and T. Weigand, Nucl. Phys. B 874, 1 (2013) arXiv:1304.2767 [hep-th]].

[61] H. Gies, S. Rechenberger, M. M. Scherer and L. Zambelli, arXiv:1306.6508 [hep-th].

[62] D. F. Litim, Phys. Lett. B 486, 92 (2000) hep-th/0005245; Phys. Rev. D 64, 105007 (2001) hep-th/0103195. 